\title{
DETERMINACIÓN DE LA PRESENCIA DE Cryptosporidium parvum y Cyclospora sp. EN CANINOS DOMÉSTICOS (Canis familiaris) EN LOS DISTRITOS DE LIMA METROPOLITANA
}

\author{
María Romero Q. ${ }^{1}$, Amanda Chávez V. ${ }^{2}$ y Eva Casas A. ${ }^{2}$
}

\section{Adstract}

The presence of Cryptosporidium parvum and/or Cyclospora sp. oocysts in the household dog population of Lima, Peru was studied. Fecal samples were collected from 185 dogs of different ages, sexes and health status (with or without diarrhea) in 30 of the 36 districts of metropolitan Lima. Samples were processed utilizing the Modified ZiehlNielsen staining technique and $25.4 \%$ were found to be positive with $C$. parvum oocyst while $4.3 \%$ were positive with Cyclospora sp. oocyst. No significant relationships were found between the presence of C. parvum and/or Cyclospora sp. and the occurrence of diarrhea, age or sex of the surveyed animals.

Key words: Dogs, Cryptosporidium, Cyclospora, zoonosis.

\section{Resumen}

El presente estudio tuvo como objetivo determinar la presencia de ooquistes de $C$. parvum y/o Cyclospora sp. en la población canina doméstica de 30 de los 36 distritos de Lima Metropolitana. Se colectaron muestras fecales de 185 perros de diferentes edades, sexo y estado sanitario (con y sin diarrea). Las heces fueron procesadas mediante la técnica de coloración de Ziehl-Nielsen modificado; encontrándose el 25.4\% de la población positivo a ooquistes de C. parvum y el $4.3 \%$ positivo a ooquistes de Cyclospora sp. No se encontró una relación significativa entre la presencia de C. parvum y/o Cyclospora $s p$. con cuadros diarreicos, edad ni sexo en los animales muestreados.

Palabras clave: Perros, Cryptosporidium, Cyclospora, zoonosis.

\section{Timuluewtor}

La criptosporidiosis y ciclosporidiosis son enfermedades reportadas en diversos países del mundo. Las poblaciones en riesgo son personas inmunocompetentes e inmunodeprimidos de todas las edades

\section{Práctica privada}

2 Laboratorio de Parasitología IVITA - FMV UNMSM.E.mail:d170010@unmsm.edu.pe.
(Ortega, 1996; Ortega et al., 1993).

La criptosporidiosis es ocasionada por el C. parvum, reconocido como el principal causante de la diarrea en humanos y animales domésticos. (Martins y Guerrant, 1995). La ciclosporidiosis es causada por Cyclospora sp., reportada en reptiles, roedores y serpientes (Ooi et al., 1995; Ortega et al.,1993; Soave, 1996). En el hombre es reconocida como Cyclospora cayetanensis, considerada como causante 
de infecciones entéricas (Ortega et al., 1994; Colomina y Villar, 1997). Ambos agentes causan en el hombre un cuadro diarreico y prolongado (Connor, 1997), siendo difícil la diferenciación clínica y de laboratorio a menos que se realice la medición microscópica del tamaño del parásito (Brennan et al., 1996; Ooi et al, 1995; Ponce-Marcotela et al., 1996; Ortega et al., 1994).

En los últimos diez años se ha incrementado el número de infecciones humanas por criptosporidiosis. Las personas en contacto con animales y personal médico en general, fueron los que presentaron un mayor número de infecciones (Kirk y Bonagura, 1994). Estas parasitosis son transmitidas mediante la ingestión de agua y alimentos contaminados con ooquistes de C. parvum y/o Cyclospora sp.; presentándose la infección en los meses de mayor temperatura (Ortega, 1996; Guerrant, 1997; Soave et al., 1998; Min. Salud, 1998). El $7 \%$ de las diarreas neonatales en el Perú, están asociadas con la criptosporidiosis (Castillo et al., 1993); asimismo, se ha reportado en el $11 \%$ de niños de 0 a 2.5 años de edad (Madico et al., 1997).

En otros países, la criptosporidiosis ha sido reportada en cachorros, perros enfermos con distémper y raramente en perros adultos (Kirk y Bonagura, 1994). En nuestro medio, existe sólo un estudio sobre criptosporidiosis en animales de compañía, realizado por Fernández (1998), quien reportó $6.98 \%$ de $C$. parvum en gatos domésticos de Lima Metropolitana.

El perro es uno de los animales de compañía más preferido por nuestra sociedad por la facilidad de compartir los ambientes físicos de la casa, y llega a tener un contacto muy cercano con los niños. Es así, que las posibilidades de transmisión de enfermedades del perro al hombre, incluyendo la criptosporidiosis y la ciclosporidiosis son bastante altas. Por tal razón, se planteó el presente estudio, a fin de determinar la presencia de Cryptosporidiun parvum y/o Cyclospora sp. en los perros domésticos de los distritos de Lima Metropolitana.

\section{Materiales y Netudos}

El muestreo se realizó en 30 de los 36 distritos que comprende Lima Metropolitana, durante el período de setiembre de 1998 a junio de 1999. El tamaño de muestra fue de 185 perros seleccionados aleatoriamente, luego de la estratificación por distrito según la población estimada. El tamaño muestral se determinó mediante la fórmula para estimación de una proporción, con $95 \%$ de confianza y $5 \%$ de precisión, con una prevalencia de $7.4 \%$ (Causape et al., 1996).

La toma de muestra se realizó de consultorios veterinarios y casas particulares, evitándose tomar más de una muestra por casa o por consultorio a fin de evitar un sesgo en el muestreo. Se obtuvo información sobre la edad y sexo de los animales, así como la presencia de heces acuosas o no sólidas, denominándose como diarreicas.

Las muestras se colectaron en forma individual en bolsas de plástico individuales, obteniéndose aproximadamente 5 $\mathrm{g}$ de heces, las mismas que fueron convenientemente identificadas y almacenadas en refrigeración hasta su procesamiento en el Laboratorio de Parasitología de la Facultad de Medicina Veterinaria de la UNMSM. Se realizaron dos frotices por muestras, los que fueron fijados en metanol absoluto por cinco minutos. Posteriormente se colorearon con el método de Ziehl Nielsen Modificado y se observaron al microscopio de luz a un aumento de 400X para la ubicación y $1000 \mathrm{X}$ en inmersión para el diagnóstico. Se 
consideraron positivo los frotices de heces que presentaban organismos de color rojo fucsia y esférico, en un fondo verde como contraste, compatibles a $C$. parvum (4 a 6 $\mu$ de diámetro) y a Cyclospora sp. (10.8 $\mu$ de diámetro).

Se evaluó la asociación entre la ocurrencia de $C$. parvum y/o Cyclospora sp. con la edad (cuatro grupos de edad), sexo (macho y hembra) y la presencia o ausencia de diarrea mediante la prueba de Chi cuadrado.

\section{Resultadiss}

De las 185 muestras analizadas correspondientes a los cuatro grupos etarios, se encontró un $25.4 \%$ de muestras positivas a $C$. parvum y $4.3 \%$ a Cyclospora $s p$ (Cuadro 1). La distribución de animales positivos a $C$. parvum y Cyclospora $s p$. con o sin cuadros diarreicos no mostró diferencia significativa (Cuadro 2).

\section{Disension}

La mayoría de trabajos realizados sobre la criptosporidiosis concluyen que es una enfermedad de mayor presentación en animales jóvenes (Fernández et al., 1988; Ortega, 1996). De la misma manera trabajos realizados en gatos de Lima Metropolitana por Fernández (1998); demuestran un alto porcentaje de animales positivos (11.4\%) en el grupo de edad de 0 a 6 meses. Asimismo, en estudios realizados en alpacas menores de 15 días y en terneros de 1 a 3 semanas de edad se reportan prevalencias más altas que animales de más edad, (Fernández, 1995; Morales, 1996). Sin embargo, en el presente estudio esta conclusión no se cumple, al encontrarse una frecuencia total de animales positivos a $C$. parvum de $25.4 \%$, no habiéndose hallado diferencias significativas entre los grupos de edad.

Nuestro hallazgo difiere de los reportados en perros de las ciudades de California, USA y Zaragoza, España, en los cuales utilizando técnicas de diagnóstico similares reportaron prevalencias de 2 y 7\% respectivamente (El-Ahraf et al., 1991; Causape et al., 1996). La cifra considerablemente alta de este trabajo, puede deberse a diferentes factores como, la presencia en nuestro medio de animales con bajo nivel nutricional, inmunodeficientes $o$ inmunodeprimidos, entre otros (Kirk y Bonagura, 1994). Asimismo, debemos considerar que en ciudades de los Estados Unidos de Norte América y Europa no está permitida la circulación de perros vagos. En el caso de Lima Metropolitana, la mayoría de los perros son alimentados con excedente de comida casera, por lo que su dieta puede considerarse deficiente o desbalanceada; disponiendo además de un deficiente control sanitario, aumentando su riesgo a sufrir enfermedades parasitarias, bacterianas o virales.

El relación a la Cyclospora sp. se determinó un $4.3 \%$. (8/185) de perros infectados en Lima Metropolitana, con una mayor frecuencia de animales positivos en el grupo de 0 a 6 meses de edad (6.2\%). Este hallazgo es el primero realizado en perros de nuestro medio, encontrándose en la literatura consultada el hallazgo de Cyclospora sp. realizado en dos perros de Sao Paulo (Yai et al., 1997). Es posible que esta enfermedad sea, de mayor presentación en animales jóvenes al igual que lo que sucede con la criptosporidiosis (Fernández, 1998). 
Cuadro 1. Frecuencia de animales positivos a Cryptosporidium parvum y Cyclospora sp., según edad en perros domésticos de Lima Metropolitana.

\begin{tabular}{cccc}
\hline Edad (meses) & $\begin{array}{c}\text { Perros, } \\
\mathrm{n}\end{array}$ & $\begin{array}{c}\text { C. } \text { parvum }^{1}, \\
\mathrm{n}\end{array}$ & $\begin{array}{c}\text { Cyclospora sp. }^{2}, \\
\mathrm{n}\end{array}$ \\
\hline $0-6$ & 80 & 24 & 5 \\
$7-12$ & 27 & 6 & 0 \\
$13-72$ & 53 & 11 & 2 \\
$>72$ & 25 & 6 & 1 \\
\hline Total & 185 & 47 & 8 \\
\hline
\end{tabular}

$\pi, 2$ : No significativo

Cuadro 2. Frecuencia de animales positivos a Cryptosporidium parvum y Cyclospora $s p$., según presentación de diarrea en perros domésticos de Lima Metropolitana.

\begin{tabular}{lcccccc}
\hline Condición & \multirow{2}{*}{ Perros, $\mathrm{n}$} & \multicolumn{2}{c}{ C. parvum $^{\mathrm{l}}$} & \multicolumn{2}{c}{ Cyclospora sp. ${ }^{2}$} \\
\cline { 3 - 6 } & & $\mathrm{n}$ & $\% \pm \mathrm{I}$.C. & $\mathrm{n}$ & $\% \pm \mathrm{I} . \mathrm{C}$ \\
\hline Con Diarrea & 92 & 23 & $25.0 \pm 8.8$ & 6 & $6.5 \pm 5.0$ \\
Sin Diarrea & 93 & 24 & $25.8 \pm 8.9$ & 2 & $2.1 \pm 2.9$ \\
\hline Total & 185 & 47 & $25.4 \pm 6.3$ & 8 & $4.3 \pm 2.9$ \\
\hline${ }^{1.2}:$ No significativo & & & & &
\end{tabular}

La poca relación existente entre cuadros diarreicos y la presencia de $C$. parvum indicaría que el perro llega a infectarse con Cryptosporidium, sin desarrollar la enfermedad. Resultados similares fueron reportados por Lloyd y Smith (1997) en perros, donde se logró producir la infección, mas no la enfermedad. Debido al escaso número de animales positivos a Cyclospora sp. no podemos concluir la existencia o no de relación de la presencia de cuadros diarreicos y el hallazgo de este parásito. No se observó relación alguna entre presencia de $C$. parvum y Cyclospora sp, y el sexo de los animales (Cuadro 3).
El presente estudio estuvo enfocado en demostrar la presencia del C. parvum, así como la Cyclospora sp. en perros de Lima Metropolitana, sin intentar comparar diferencias entre perros por distrito, tipo de crianza (con propietario vs. perros vagos), etc. Sin embargo, y considerando los resultados encontrados, se hace necesario realizar estudios posteriores al respecto; así como tratar de confirmar la tesis de Georgi y Georgi (1994), quienes propusieron que los perros pueden actuar como portadores asintomáticos a C. parvum. Por otro lado, los resultados obtenidos podrían indicar que los perros aparentemente sanos pueden actuar como una fuente de infección 
al hombre (El-Ahraf et al., 1991; Causape et al., 1996). Finalmente, cabe indicar que se desconocen muchos aspectos de la ciclosporidiosis, tales como los mecanismos por los cuales estos parási- tos causan enfermedad, relación de hospederos, así como sus reservorios (Soave et al., 1998) de allí la conveniencia de profundizar los estudios en estas áreas.

Cuadro 3. Frecuencia de animales positivos a Cryptosporidium parvum y Cyclospora $s p$., según sexo en perros domésticos de Lima Metropolitana.

\begin{tabular}{lccccc}
\hline Sexo & Perros, & \multicolumn{2}{c}{${\text { C. } \text { parvum }^{1}}^{2}$} & \multicolumn{2}{c}{ Cyclospora sp. $^{2}$} \\
\cline { 3 - 6 } & $\mathrm{n}$ & $\mathrm{n}$ & $\% \pm$ I.C. & $\mathrm{n}$ & $\% \pm$ I.C. \\
\hline Machos & 97 & 28 & $28.9 \pm 9.0$ & 5 & $5.2 \pm 4.4$ \\
Hembras & 88 & 19 & $21.6 \pm 8.6$ & 3 & $3.4 \pm 3.8$ \\
\hline Total & 185 & 47 & $25.4 \pm 6.3$ & 8 & $4.3 \pm 2.9$ \\
\hline
\end{tabular}

${ }^{12}:$ No significativos

\section{Titeritura Clomb}

\section{Brennan, M.K.; D.W. Mac Pherson;} J. Palmer y J.S. Keystone. 1996. Cyclosporidiasis: a new cause of diarrhea. Can. Med. Assos. J. 155 (9): $129-126$.

2. Castillo, R.; F. Diaz; E. Miranda; $R$. Gilmam; J. Naranjo y grupo de Cistecercosis. 1993. Detección de Anticuerpos (Ig. G) contra Criptosporidium por western blot. Res. III Cong. Lat Parasitol. Lima, Perú: 166.

3. Causape, A.C.; J. Quillez; C. Sánchez-Acedo y E. Del Cacho. 1996. Prevalence of intestinal parasites, including Cryptosporidium parvum, in dogs in Zaragoza city, Spain. Vet. Parasitol. 63 (3-4): 161-167.

4. Colomina, J.R. y J.S. Villar. 1997. Morphological, clinical and therapeutic characteristics of Cyclospora cayatensis. Bol. Chil. Parasitol. 52 (12): 26-32.

5. Connor, B.A. 1997. Cyclospora infection: a review. Anm. Acad. Med. Singapore. 26 (5): 632-636.

6. El-Ahraf, A.; J.V.Tacal; M. Sobih; W. Lawrence y B.W. Wilcke. 1991. Prevalence of Criptosporidiosis in dogs and human beings in San Benardino county, California. J.A.V.M.A. 198 (4). 631-634.

7. Fernández, A.J.; J. Pohlez; M.A. Sierra y A. Jover. 1988. Criptosporidiosis. Med. Vet. 5 (12): 615-28.

8. Fernández, F.M. 1995. Prevalencia de Cryptosporidium parvum en alpacas neonatas del centro experimental $\mathrm{La}$ Raya, Cuzco. Tesis Médico Veterinario. Facultad de Medicina Veterinaria. UNMSM. 38p.

9. Fernández, P.V. 1998. Detección de Crytosporidium parvum en felinos domésticos de Lima Metropolitana. Tesis Médico Veterinario. Facultad de Medicina Veterinaria. UNMSM. 47 p. 10. Georgi, J.R. y M.E. Georgi. 1994. Parasitología en clínica canina. 1ra. Ed., 
p:86. Editorial Interamericana, México.

11. Guerrant, R.L. 1997. Criptosporidiosis: An emerging highly infectious threat. Emer. Inf. Dis. 3 (1): 51-57.

12. Kirk, R.W. y J.D. Bonagura. 1994. Terapeútica veterinaria de pequeños animales. 11 Ed.p:316-322. Editorial Interamericana, España.

13. Lloyd, S. y J. Smith 1997. Pattern of Crytosporidium parvum oocyst excretion by experimentally infected dogs. Int. J. Parasitol. 27 (7): 799-801.

14. Madico,G.; J. McDonal; R.H. Gilman; L. Cabrera y C.R. Sterling. 1997. Epidemiology and treatment of Cyclospora cayatanensis infection in peruvian children. Clin. Inf. Dis. 24 (5): 977-81.

15. Martins, C.A.P. y R.I. Guerrant 1995 Crytosporidium and Criptosporidiosis. Parasitol. Today. 11(1): 434-436.

16. Ministerio de Salud. 1998. Parásitos oportunistas y asociados al SIDA. Laboratorio de referencia de enteroparásitos.

17. Morales, H.M.1996. Prevalencia de criptosporidiosis en alpacas neonatas en el Departamento de Puno. Tesis Médico Veterinario. Facultad de Medicina Veterinaria. UNMSM. $40 \mathrm{p}$.

18. Ooi, W.W.; S.K. Zimmerman y C.A. Needham. 1995. Cyclospora species as a gastrointestinal pathogen in inmunocompetent hosts. J. Clin. Microb. 33 (5): 1267-1269.

19. Ortega, L.M. 1996. Biología, epidemiología y control de criptosporidiosis. Universidad Complutense de Madrid: Facultad de Medicina Veterinaria. (7):9.

20. Ortega, Y.R.; C.R. Sterling; R.H. Gilman; V.A. Cama y F. Díaz. 1993 Cyclospora species. A new protozoan pathogen of humans. New Engl. J. Med. 328: 1308-1312.

21. Ortega, Y.R.; R.H. Gilman y C.R. Sterling 1994. A new coccidian parasite (Apicomplexa: Eimeriidade) from humans. J. Parasitol. 80 (4): 625 629.

22. Ponce-Marcotela, M.; C. Cob-Soza y M.N. Martinez-Gordillo. 1996. Cyclospora in 2 Mexican children. Rep. Inv. Clin. 48(6):461-463.

23. Soave, R. 1996. Cyclospora: An overview. Clin. Inf. Dis. 23: 429-435.

24. Soave, R.; B.L. Herwaldt y D.A. Relman. 1998. Cyclospora. Inf. Dis.Clin. North. Am. 12 (1): 1-12.

25. Yai, L.E.; A.R. Bavab; M.P. Hirschfeld; M.L. De Olivera y J.T. Damacdeno. 1997. The first two cases of Cyclospora in dogs, Sao Paulo, Brazil. Rev. Inst. Med. Trop. Sao Paulo. 39 (3):177-179. 